\title{
Constant versus variable response signal delays in speed-accuracy trade-offs: Effects of advance preparation for processing time
}

\author{
JEFF MiLleR \\ University of Otago, Dunedin, New Zealand \\ AND \\ GUDRUN SPROESSER AND ROLF ULRICH \\ University of Tübingen, Tübingen, Germany
}

\begin{abstract}
In two experiments, we used response signals (RSs) to control processing time and trace out speed-accuracy trade-off (SAT) functions in a difficult perceptual discrimination task. Each experiment compared performance in blocks of trials with constant and, hence, temporally predictable RS lags against performance in blocks with variable, unpredictable RS lags. In both experiments, essentially equivalent SAT functions were observed with constant and variable RS lags. We conclude that there is little effect of advance preparation for a given processing time, suggesting that the discrimination mechanisms underlying SAT functions are driven solely by bottom-up information processing in perceptual discrimination tasks.
\end{abstract}

A fundamental characteristic of human performance is the ability to trade speed for accuracy (Pachella, 1974). In virtually all perceptual, cognitive, and motor tasks, people can choose to respond relatively quickly and produce responses with relatively low accuracy, or they can choose to respond more slowly and achieve greater accuracy. Many researchers have studied these speed-accuracy trade-offs (SATs) in order to characterize the inherent flexibility of the mechanisms underlying human performance.

Two experimental procedures that have often been used to study SATs are the deadline procedure and the response signal (RS) procedure. In the deadline procedure, participants are told in advance of each trial to respond within a certain prespecified time after stimulus onset - that is, to respond quickly enough to beat a deadline (e.g., Green \& Luce, 1973; Lien, Ruthruff, Remington, \& Johnston, 2005; Link \& Tindall, 1971; Pachella, Fisher, \& Karsh, 1968; Ratcliff \& Rouder, 2000; for reviews, see Luce, 1986; Pachella, 1974; Wood \& Jennings, 1976). People are remarkably good at generating responses just before the deadline has elapsed (Pachella \& Pew, 1968), and their response accuracy increases monotonically with the length of the deadline. Thus, the deadline procedure allows researchers to manipulate participants' processing time and trace out empirical SAT functions like the one shown in Figure 1. This procedure can even be used in experiments in which the prespecified deadline varies randomly from trial to trial (e.g., Gopher, Armony, \& Greenshpan, 2000; Kleinsorge, 2001; Link, 1971; but see Strayer \& Kramer, 1994b).
An alternative to the deadline procedure is the RS procedure, in which participants are given an explicit signal at the moment at which they must respond (e.g., Corbett \& Wickelgren, 1978; Dosher, 1976, 1982; Dosher, Han, \& Lu, 2004; McElree \& Carrasco, 1999; Ratcliff \& McKoon, 1989; Reed, 1973, 1976; Wickelgren \& Corbett, 1977). Reed (1973) introduced this procedure with a recognition memory experiment in which a single probe letter was presented in each trial for an old/new judgment. The probe letter was displayed for $0.5,1,2,4$, or $8 \mathrm{sec}$, and the participants were told to respond immediately at the offset of the probe letter. Letter offset thus served as the RS in this experiment, although auditory RSs have been more common in subsequent experiments with visual test stimuli (e.g., Dosher, 1976). The experimenter-controlled time from the onset of the test stimulus to the RS is known as the RS lag. After some practice, participants can fairly consistently respond within approximately $200-250 \mathrm{msec}$ of the RS, so the RS lag effectively controls the amount of time used to perform the task on a given trial. Like the deadline procedure, then, the RS procedure allows researchers to manipulate participants' processing time, thereby tracing out empirical SAT functions of the form shown in Figure 1.

Although both the deadline and the RS procedures are widely used for tracing out empirical SAT functions, there are several clear and potentially important differences between them. For example, the RS procedure necessarily involves the presentation not only of the main task-relevant

J. Miller, miller@psy.otago.ac.nz 


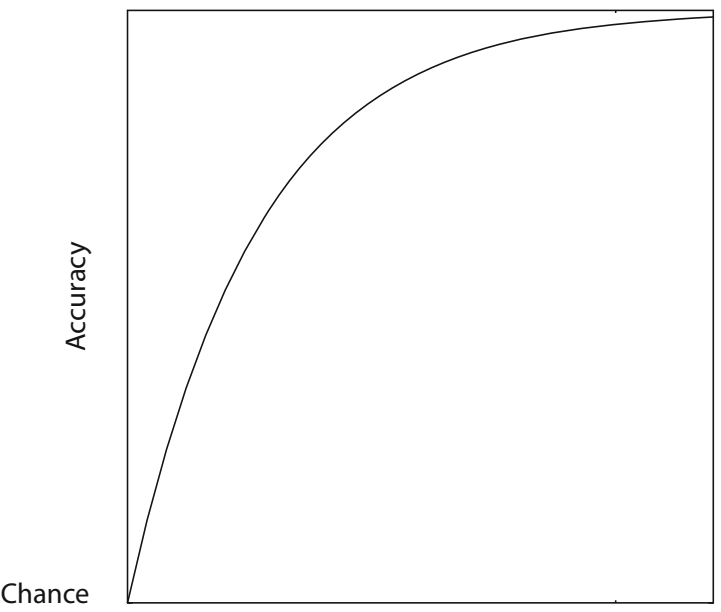

Reaction Time

Figure 1. Idealized speed-accuracy trade-off function tracing out the relationship between reaction time and response accuracy.

stimulus, but also of an auxiliary stimulus telling the participant when to respond. Relative to the deadline procedure in which no RS is presented, this auxiliary stimulus may increase arousal in the RS procedure (e.g., Low, Larson, Burke, \& Hackley, 1996; Stoffels, van der Molen, \& Keuss, 1985), or it may decrease the amount of attention available for processing the main task (e.g., Bonnel \& Hafter, 1998; Massaro \& Warner, 1977; Tulving \& Lindsay, 1967).

In this work, we focus on a specific difference between the deadline and the RS procedures that seems particularly important for our understanding of the mechanisms of strategic adjustment involved in SATs - specifically, advance knowledge of processing time. In the deadline procedure, the participant knows in advance how much time will be available to perform the task and can therefore try to respond as accurately as possible in that prespecified amount of time. In the RS procedure with randomized lags, in contrast, the participant does not know how much time is available for processing until that time is over. In this procedure, the participant must be ready to respond as accurately as possible in whatever amount of time happens to be made available. In short, these two procedures differ with respect to advance knowledge of the time that will be available for carrying out the task.

This article reports two experiments conducted to explore the consequences, if any, of this difference in advance knowledge. To isolate this difference in advance knowledge, we modified the usual deadline procedure slightly by presenting an RS in each trial-just as in the RS procedure - but using a constant RS lag for all the trials within a block. For brevity, we will refer to this modified deadline procedure as the deadline procedure, although we acknowledge that it deviates in this way from the deadline procedure as it has most often been used in previous studies.

One obvious question is whether the efficiency of speeded performance depends on advance knowledge of the time available for processing. If so, one would presumably expect better performance in the deadline procedure, for at least three interrelated reasons. First, in the deadline procedure, participants may be able to use advance information to select a processing strategy that will be optimal for the available time. Participants can also adopt special processing strategies with the RS procedure, of course, but these cannot be optimized for the available time because this time is not known until processing must be terminated. Indeed, early proponents of the RS procedure favored it over the deadline procedure partly because it eliminated the possibility that processing strategy might vary with processing time (Dosher, 1976; Reed, 1976; cf. Ratcliff, 2006), since there is evidence that it does (e.g., Dror, Busemeyer, \& Basola, 1999). Second, the deadline procedure clearly allows better temporal preparation than does the RS procedure. It has repeatedly been demonstrated that participants in a reaction time (RT) task can prepare more efficiently for the onset of an imperative stimulus when the interval between a warning signal and the succeeding imperative stimulus is constant across trials than when it varies randomly from trial to trial (e.g., Niemi \& Näätänen, 1981). As a result, constant foreperiods yield shorter RTs than do variable ones, at least for foreperiods longer than approximately $300 \mathrm{msec}$ (Bertelson \& Tisseyre, 1968). Obviously, participants can more easily anticipate the imperative stimulus in the constant condition, which enables faster (e.g., Mattes \& Ulrich, 1997) and more accurate (e.g., Rolke \& Hofmann, 2007) processing of stimulus information. Such foreperiod effects suggest that uncertainty about when the RS will be presented may reduce performance in the RS procedure relative to the deadline procedure, at least for RS lags longer than about $300 \mathrm{msec}$. A third and related point is that RTs to the RS may be inflated by the requirement to detect and respond to this temporally unexpected stimulus, particularly if more attentional resources must be withheld from task processing in order to monitor for the occurrence of an unpredictable $\mathrm{RS}$.

If performance is better with the deadline procedure than with the RS procedure, as the preceding three reasons suggest, it seems likely that this performance difference should be especially large when the RS lag is short. In general, relatively short lags minimize processing time and should, therefore, be most sensitive to differences in processing efficiency. In contrast, at long RS lags, accuracy can, in principle, approach the same asymptotic level regardless of efficiency, because even relatively inefficient processing will eventually produce the correct result. According to this view, the SAT function will first increase above-chance performance (cf. Figure 1) at an earlier time point with the deadline procedure than with the RS procedure. Participants may adopt a crude but fast strategy when they know that a fast response will be required (i.e., in the deadline procedure with a short deadline), and that strategy can lead to (say) $60 \%-70 \%$ correct responses. With the RS method, they may, instead, adopt a slower strategy that will lead to much higher accuracy levels when more processing time is available, but this strategy may produce chance performance on occasional trials when the lag is very short. 
As one example, suppose that participants are required to discriminate between long and short lines. A possible crude but fast strategy may be to respond on the basis of an overall brightness judgment, rather than analyzing length directly. This strategy may lead to better-than-chance discrimination if longer lines tend to be perceived as brighter, on average, than shorter ones, even though it may not be as accurate in the long run as focusing on length per se. As another example, suppose that (1) participants can potentially combine information from several cues in order to reach an optimal judgment (see Lee \& Cummins, 2004) and (2) the different cues take different amounts of time to process, with time not particularly correlated with the diagnosticity of the cue. If participants know how much time will be available for the judgment, they can process the optimal set of cues to get the best information in the time available. In contrast, if the available processing time is unknown, participants can process the cues only in an order that will be optimal on average across processing times, not for each particular processing time.

The question of whether advance knowledge of processing time has any influence on empirical SAT functions seems important both theoretically and methodologically. Theoretically, if performance really is better with deadlines than with RSs, it would follow that advance knowledge must allow useful strategic adaptation based on the amount of time available for processing. This would imply that settings of the processes involved in generating SAT functions can be adjusted or tuned in advance to operate somewhat differently, depending on the amount of time to be used, and that different settings are optimal for different processing times. Alternatively, if performance is no better with deadlines than with RSs, it would appear that the processes involved in generating SAT functions are relatively strategy-invariant, bottom-up perceptual accumulation processes. These processes can be conceptualized as passive information accumulators for which only the time of accumulation matters - not any strategic variation in the accumulation process itself.

Methodologically, any differences in results obtained with these two procedures will clearly be of interest for researchers choosing which procedure to use in tracing out desired SAT functions. One obvious question, for example, is which procedure provides better experimental control over RT. Ideally, researchers studying SATs would like to be able to control the mean RT in a given condition, and they would like to do that while keeping within-condition RT variability relatively low, so that participants take the appropriate amount of time not only on average, but also on each individual trial. Thus, it is of methodological interest to compare the observed mean and within-condition standard deviations produced by the deadline and RS methods.

To address these theoretical and methodological issues, we carried out two experiments investigating whether advance knowledge about the time available for processing affects the efficiency of speeded information processing. Specifically, we compared SAT functions traced out with versus without advance knowledge of the available processing time, to see whether these functions would be superimposed. To equate the stimulus events as closely as possible across conditions with versus without advance knowledge, we used the RS paradigm as mentioned earlier, with an identical auditory RS presented on each trial to indicate when the participant should respond. To create conditions with versus without advance knowledge, we compared blocks of trials on which the RS lag was constant against blocks in which this lag varied randomly. ${ }^{1}$

\section{EXPERIMENT 1}

The present experiments used the two-alternative forced choice line length discrimination task studied by Rinkenauer, Osman, Ulrich, Müller-Gethmann, and Mattes (2004). This task was chosen because it had been found to give a relatively gradual increase in accuracy as a function of RT, which presumably would maximize power in detecting an effect of advance knowledge on the SAT function. On each trial, a cross consisting of a vertical and a horizontal line was presented in the center of a computer screen. The horizontal line was always either slightly above or slightly below the middle of the vertical line. Participants had to respond immediately after the presentation of an auditory RS with a left-hand or righthand keypress to indicate whether the upper or the lower segment of the vertical line was longer. On different trials, the RS lag (i.e., the time from the onset of the stimulus to that of the RS) was $75,175,300$, or $450 \mathrm{msec}$.

The primary experimental comparison involved different types of blocks in which the participants did versus did not know in advance the amount of time that would be available for processing. In constant blocks, the RS lag was the same on every trial, so the participants knew in advance of the trial when the RS would be presented (i.e., when the response would be required). In variable blocks, in contrast, the different RS lags were randomly intermixed within a single block, so the amount of time available for processing was not predictable in advance of the trial.

\section{Method}

Participants. Eight right-handed students ( 5 of them female) were tested individually in five 1-h sessions held at approximately the same time on consecutive days. Their mean age was 21.9 years $(S D=$ 2.1 years). Four participated as volunteers, and 4 were paid NZ \$50.

Apparatus. Stimulus presentation was controlled by an IBMcompatible PC. Visual stimuli were presented as white figures on the dark background of a computer monitor that was viewed from a distance of approximately $60 \mathrm{~cm}$, and auditory stimuli were presented binaurally via headphones. The participants were tested individually in a dimly illuminated cubicle, and they responded by pressing the "Z" and "?" keys of the computer keyboard with their left and right index fingers, respectively.

Stimuli and Task. Each trial began with the presentation of a fixation point in the middle of the screen for $500 \mathrm{msec}$. At the offset of the fixation, a horizontal line subtending approximately $0.5^{\circ}$ appeared, centered at the position of the fixation. After $500 \mathrm{msec}$, a vertical line $\left(5.7^{\circ}\right)$ was presented for $50 \mathrm{msec}$, making a cross with the horizontal line. The vertical line bisected the horizontal line, but the top part of the vertical line was either shorter or longer than the bottom part by 2 pixels (approximately $0.1^{\circ}-0.2^{\circ}$ ). Finally, the RS was an $800-\mathrm{Hz}$ tone $(100 \mathrm{msec}$ in duration, approximately $50 \mathrm{~dB}$ ), presented binaurally over headphones at one of four RS lags $(75,175,300$, or $450 \mathrm{msec})$ after the onset of the vertical line. 
Half of the participants were instructed to respond with the left hand when the top part was longer and with the right hand when the bottom part was longer. For the other half of the participants, these response assignments were reversed. All the participants were instructed to respond immediately after the tone was presented, and they were given error feedback if their responses were too early or too late. Specifically, if they did not respond within a grace period of $300 \mathrm{msec}$ after the onset of the RS, the computer displayed a visual error message that the response had been too late, and this message remained on the screen for $5 \mathrm{sec}$. Responses before the onset of the RS were also discouraged with a 5-sec message saying that the response had been too early and reminding the participant to wait for the RS before responding. The long duration of these messages was intended to motivate the participants to respond at the signaled time. In contrast, when the participants responded within $0-300 \mathrm{msec}$ after the RS but responded incorrectly, the word ERROR appeared for only $1 \mathrm{sec}$.

Procedure. Within each session, each participant was tested in 16 blocks, with 40 trials per block, with forced 2-min rest breaks after Blocks 4,8 , and 12 . In 8 consecutive blocks, RS lag varied randomly, with each of the four lags tested equally often in each block. In the other 8 consecutive blocks, RS lag was constant, with 2 constant blocks at each of the four lags. The order of the blocks was counterbalanced across sessions for each participant. Half of the sessions started with the 8 randomized blocks, and the other half started with the 8 constant blocks. The 2 constant blocks with a given RS lag were always run consecutively, and the order of the four constant-block pairs within the 8 constant-lag blocks was varied across sessions according to a Latin square. In all the blocks, the upper half of the vertical line was longer than the lower half on half of the trials at each of the tested RS lags.

On each trial, response accuracy was recorded, as was the RT measured from the onset of the vertical line until the keypress. The time from the onset of the RS to the keypress was computed as $\mathrm{RT}_{\mathrm{RS}}=\mathrm{RT}-$ lag, and this variable was checked to make sure that the participants responded in the appropriate time window after RS onset. Thus, the total RT interval can be conceived of as the sum of the RS lag and $\mathrm{RT}_{\mathrm{RS}}$.

\section{Results}

We analyzed the RTs for both the correct responses and errors, but we excluded trials on which the participant responded before the onset of the RS $(0.3 \%)$ or more than $400 \mathrm{msec}$ after it $(0.8 \%)$.

Basic findings. For each combination of RS lag, constant versus variable block, stimulus type (i.e., upper vs. lower line longer), and participant, we computed the percentage of correct responses (PC), the means of RT and $\mathrm{RT}_{\mathrm{RS}}$, and the standard deviation of $\mathrm{RT}$ and $\mathrm{RT}_{\mathrm{RS}}(S D)$, pooling across Sessions 2-5 for each participant. ${ }^{2}$ Table 1 shows the averages of these values across participants and stimulus types.

Repeated measures ANOVAs were carried out for each of the four dependent variables shown in Table 1, using factors of RS lag and block type. As was expected, RS lag had highly significant effects on three of the four measures, with PC increasing at longer lags $[F(3,21)=$ $\left.260.72, M S_{\mathrm{e}}=21.01, p<.001\right], \mathrm{RT}$ increasing at longer lags $\left[F(3,21)=1,655.60, M S_{\mathrm{e}}=315.63, p<.001\right]$, and $\mathrm{RT}_{\mathrm{RS}}$ decreasing at longer lags $\left[F(3,21)=124.52, M S_{\mathrm{e}}=\right.$ $315.63, p<.001] .{ }^{3}$ The difference between constant and variable blocks was significant for all four measures. PC was higher in variable blocks than in constant ones $\left[F(1,7)=18.61, M S_{\mathrm{e}}=16.39, p<.005\right]$. In addition, responses were slower in variable blocks than in constant ones for both RT $\left[F(1,7)=22.12, M S_{\mathrm{e}}=546.55, p<\right.$ $.005]$ and $\mathrm{RT}_{\mathrm{RS}}\left[F(1,7)=22.12, M S_{\mathrm{e}}=546.55, p<.005\right]$. Finally, $S D$ was smaller in variable blocks than in constant ones $\left[F(1,7)=8.16, M S_{\mathrm{e}}=39.12, p<.025\right]$. Theoretically, however, it is difficult to see why RTs would have a higher variance when participants know in advance what RT will be required (constant blocks) than when they do not know in advance (variable blocks), but that is what these data indicate.

Most important, significant block type $\times$ lag interactions were observed, indicating that the effect of RS lag was larger in constant blocks than in variable blocks for $\operatorname{PC}[F(3,21)=$ $\left.8.57, M S_{\mathrm{e}}=12.80, p<.005\right]$ and for RT $[F(3,21)=15.64$, $\left.M S_{\mathrm{e}}=225.59, p<.001\right]$ but that it was smaller in constant blocks than in variable blocks for $\mathrm{RT}_{\mathrm{RS}}[F(3,21)=15.64$, $\left.M S_{\mathrm{e}}=225.59, p<.001\right]$. This interaction is important because it shows that the participants were affected by advance knowledge about the available processing time, rather than simply waiting for the RS in all blocks. There was also a significant interaction of block type and RS lag for $S D$ $\left[F(3,21)=3.92, M S_{\mathrm{e}}=14.304, p<.05\right]$, although this interaction was small and rather unsystematic.

On the basis of these results, the constant lag procedure has the advantage that it yields somewhat larger shifts in mean RT and PC, for a given change in lag, than does the variable lag procedure. Thus, it appears that the constant procedure, in effect, causes larger shifts in speed-accuracy criteria than does the variable procedure, although these changes may be due to processing that takes place after RS onset, as reflected in $\mathrm{RT}_{\mathrm{RS}}$. Interestingly, the presence of only a small block-related difference in $S D$ s indicates that the two procedures produce roughly comparable withincell RT variation. Theoretically, it is interesting that RTs seem to be at least as variable when participants know in advance what RT will be required (constant blocks) as when they do not know in advance (variable blocks). Evidently, the opportunity to prepare to produce a specific RT value does not reduce the sources of random fluctuation that produce RT variability.

Analysis of SAT functions. Because both RT and PC varied as a function of RS lag, overall performance measures combining RT and accuracy are needed to compare performance in constant versus variable blocks (Pachella, 1974; see Figure 1). Figure 2 shows accuracy as a function of mean RT, using $d^{\prime}$ as the measure of accuracy (e.g., Macmillan \& Creelman, 1991).

Table 1

Percentage of Correct Responses (PC), Mean Reaction Time From Stimulus Onset (RT), Mean Reaction Time

From Response Signal Onset $\left(\mathrm{RT}_{\mathrm{RS}}\right)$, and Standard Deviation of RT and $\mathrm{RT}_{\mathrm{RS}}(S D)$ As a Function of Block Type and Response Signal (RS) Lag in Experiment 1

\begin{tabular}{|c|c|c|c|c|c|c|c|c|}
\hline \multirow{3}{*}{$\begin{array}{l}\text { Dependent } \\
\text { Variable }\end{array}$} & \multicolumn{8}{|c|}{ Block Type } \\
\hline & \multicolumn{4}{|c|}{ Variable RS Lag (msec) } & \multicolumn{4}{|c|}{ Constant RS Lag (msec) } \\
\hline & 75 & 175 & 300 & 450 & 75 & 175 & 300 & 4 \\
\hline & 6 & & & & & 77 & 8 & \\
\hline $\mathrm{T}($ & 354 & 420 & 509 & 626 & 31 & 388 & 491 & \\
\hline $\mathrm{RT}_{\mathrm{RS}}$ & 279 & 245 & 209 & 176 & 242 & 213 & 191 & \\
\hline$S D$ (msec) & 41 & 39 & 37 & 41 & 44 & 44 & 41 & \\
\hline
\end{tabular}




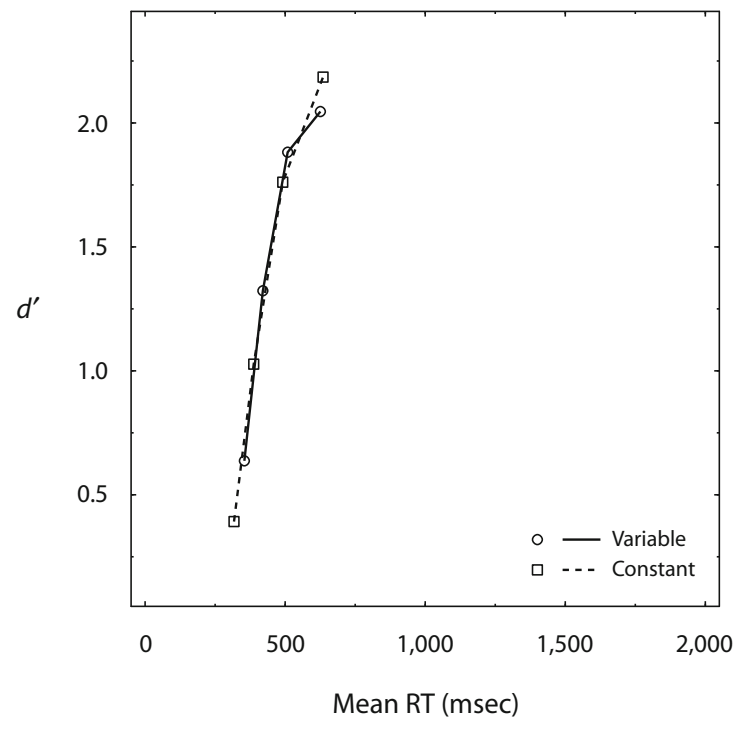

Figure 2. Response accuracy $\left(d^{\prime}\right)$ as a function of mean reaction time (RT) in constant and variable blocks in Experiment 1. The standard errors of the $d^{\prime}$ and mean RT values are 0.10 units and $6.2 \mathrm{msec}$, respectively, based on pooled error terms for both main effects and the interaction in ANOVAs with factors of block and stimulus onset asynchrony.

We summarized performance within constant and variable blocks by using a standard model in which the SAT function has the form of an exponential approach to an asymptote (e.g., Carrasco \& McElree, 2001; McElree \& Carrasco, 1999; McElree \& Dosher, 1993). Specifically, the SAT functions were fit with the three-parameter model

$$
d^{\prime}(t)=\lambda\left(1-e^{-\beta(t-\delta)}\right), \text { for } t>\delta ; \text { else, } 0,
$$

where $d^{\prime}$ is the standard signal detection measure of observed discrimination accuracy in a two-choice task (cf. Macmillan \& Creelman, 1991), $t$ is the observed mean $\mathrm{RT}$ at a given lag, $\lambda$ is the asymptotic value of $d^{\prime}, \beta$ is the rate of growth toward the asymptote, and $\delta$ is a minimum processing time below which discrimination performance is at chance (i.e., $d^{\prime}=0$ for $t \leq \delta$ ). Within this model, $\delta+$ $1 / \beta$ is a measure of total processing time, with $\delta$ representing the time from stimulus onset to the start of information accrual (e.g., sensory transmission delays) and $1 / \beta$ representing the time from the onset of information accrual until a point of near-asymptotic performance. This composite measure indexes how quickly the SAT curve rises after stimulus onset and is thus assumed to capture the overall speed of stimulus processing (Carrasco, Giordano, \& McElree, 2004).

The three parameters of the exponential growth model were estimated separately for each participant for the constant and variable blocks, minimizing the squared difference between predicted and observed values of $d^{\prime}$. Averages across participants of these parameter estimates and of the derived value $\delta+1 / \beta$ are shown in Table 2. The standard deviation of the block effect for each measure is also shown in the table in order to give an estimate of the interindividual variability in this effect. Differences between constant and variable blocks were assessed with repeated measures ANOVAs, but none of the differences approached significance ( $p>.1$ in all cases). It is clear that there was little power to detect processing time differences for $1 / \beta$ and $\delta+1 / \beta$, however, because of the large interindividual variability in the block effect on $1 / \beta$. This large variability probably resulted at least partly from difficulty in estimating this value without establishing a clear asymptote by testing at larger RT values (see Figure 2).

\section{EXPERIMENT 2}

Although the blocks with constant and variable lags seemed to produce closely overlapping SAT functions (see Figure 2), it seems hasty to conclude that there is no difference between these conditions solely on the basis of Experiment 1. In general, of course, it is important to replicate null results, taking pains to increase power in order to minimize Type II errors. Thus, Experiment 2 was designed to replicate and extend Experiment 1. We attempted to increase power first by increasing the number of experimental participants from 8 to 12 . We also attempted to increase power by strengthening the manipulation of constant versus variable blocks - specifically, by using a larger range of RS lags, including additional lags of 900 and $1,800 \mathrm{msec}$. In essence, a larger range of lags generates more uncertainty as to the time available for processing in the variable blocks, which would seem to increase the difference between constant and variable blocks. Adding these two longer lags also improved upon Experiment 1 by allowing for much more stable estimates of the parameters of the exponential growth model by assessing the asymptotic performance level more directly, and this would also tend to increase power in the comparison of parameter estimates across constant and variable blocks.

\section{Method}

The apparatus, procedure, and methods of analysis were the same as those used in Experiment 1, except as otherwise noted. The participants were 12 right-handed students ( 8 of them female) with a mean age of 26.6 years $(S D=5.6$ years). Six were students at the University of Tübingen; these were tested in Germany and received $€ 35$ for their participation. The other 6 were students at the University of Otago; these were tested in New Zealand and received NZ \$55. Each participant was tested individually in five 1-h sessions run at approximately the same time on each of 5 consecutive

Table 2

Average Parameter Estimates for the Model Represented by Equation 1 As a Function of Block Type in Experiment 1

\begin{tabular}{lccc}
\hline & \multicolumn{3}{c}{ Block Type } \\
\cline { 2 - 4 } \multicolumn{1}{c}{ Parameter } & Variable & Constant & $\begin{array}{c}S D \text { of } \\
\text { RS Lag }\end{array}$ \\
\hline$\delta(\mathrm{msec})$ & 316 & 297 & 31.73 \\
$\lambda$ & 2.46 & 3.66 & 1.89 \\
$1 / \beta(\mathrm{msec})$ & 125 & 287 & 272.97 \\
$\delta+1 / \beta(\mathrm{msec})$ & 441 & 584 & 252.84
\end{tabular}

Note-RS, response signal; $S D$, standard deviation; $\lambda$, asymptotic value of $d^{\prime}$, a unitless quantity. 
days. The German participants responded on a German keyboard by pressing the "Y" and "-" keys, which are at the same positions as the "Z" and "?" keys on the English keyboard. Six lags were tested: $75,175,300,450,900$, and $1,800 \mathrm{msec}$. On each day, each participant was tested in 13 blocks, with a forced 1-min break after the 1st block and forced 2-min breaks after the 4th, 7th, and 10th blocks. In the 1st block, no RSs were used, and the participants were simply instructed to respond as quickly and accurately as possible to the onset of each stimulus display. This block was intended to serve as a warm-up for the task. Of the remaining 12 blocks, 6 consecutive blocks used variable lags, and 6 consecutive blocks used constant lags, with 1 block at each of the six different lags. In the variable blocks there were 12 different trial types, depending on the lags and on whether the top or the bottom part was longer. Each trial type was shown four times, giving 48 trials per block. In the constant blocks, there were 2 different trial types, depending only on whether the top or the bottom part was longer. Each of these trial types was shown 24 times, again giving 48 trials per block. Whether a participant had to press the left or the right key when the top part of the cross was longer was again counterbalanced across participants. The order of constant and variable blocks was counterbalanced across days for each participant, and the order of the different lags for the constant blocks was randomized anew for each participant and session.

\section{Results}

The results were analyzed in the same manner as in Experiment 1 . We omitted the first day as practice, and we excluded trials on which the participant responded before the onset of the RS $(0.9 \%)$ or more than $400 \mathrm{msec}$ after it $(0.8 \%)$.

Basic findings. Table 3 shows the averages of PC, RT, $\mathrm{RT}_{\mathrm{RS}}$, and $S D$, averaging across participants, stimulus types, and trials.

Repeated measures ANOVAs were carried out for each of the four dependent variables shown in Table 3, using factors of RS lag and block type. As was expected, RS lag had highly significant effects on all four measures, with PC increasing at longer lags $\left[F(5,55)=203.39, M S_{\mathrm{e}}=\right.$ $48.81, p<.001]$, RT increasing at longer lags $[F(5,55)=$ $\left.35,340.48, M S_{\mathrm{e}}=547.52, p<.001\right]$, and $\mathrm{RT}_{\mathrm{RS}}$ decreasing at longer lags $\left[F(5,55)=56.64, M S_{\mathrm{e}}=547.52, p<.001\right]$. In contrast to the results in Experiment 1, $S D$ decreased at longer lags $\left[F(5,55)=29.22, M S_{\mathrm{e}}=95.50, p<.001\right]$, presumably reflecting an effect that is mainly associated with the very long lags included in this experiment.

The difference between constant and variable blocks was significant for RT $\left[F(1,11)=35.60, M S_{\mathrm{e}}=447.91\right.$, $p<.001], \mathrm{RT}_{\mathrm{RS}}\left[F(1,11)=35.60, M S_{\mathrm{e}}=447.91, p<\right.$ $.001]$, and $S D\left[F(1,11)=30.06, M S_{\mathrm{e}}=55.99, p<.001\right]$, with larger RTs and $\mathrm{RT}_{\mathrm{RS}} \mathrm{S}$ in variable blocks than in constant blocks, but with smaller $S D$ s in variable blocks.

As in Experiment 1, the effects of RS lag were larger in constant blocks than in variable blocks for PC $[F(5,55)=$ $\left.4.80, M S_{\mathrm{e}}=11.86, p<.010\right]$ and for RT $[F(5,55)=$ $\left.32.66, M S_{\mathrm{e}}=112.42, p<.001\right]$, but these effects were smaller in constant blocks than in variable blocks for $\mathrm{RT}_{\mathrm{RS}}$ $\left[F(5,55)=32.66, M S_{\mathrm{e}}=112.42, p<.001\right]$ and for $S D$ $\left[F(5,55)=3.21, M S_{\mathrm{e}}=27.02, p<.05\right]$.

Notably, as in Experiment 1, the constant lag procedure yielded somewhat larger changes in mean RT and $\mathrm{PC}$ than did the variable lag procedure, again suggesting that the constant procedure causes larger shifts in speedaccuracy criterion for a given range of lags. Also, as in Experiment 1, RTs were actually somewhat less variable in the variable blocks than in the constant blocks.

Analysis of SAT functions. Figure 3 shows $d^{\prime}$ as a function of mean RT, and the SAT functions for the constant and variable blocks were again clearly superimposed. We again summarized performance within constant and variable blocks in terms of an exponential approach to an asymptote (i.e., Equation 1), and the average parameter estimates are shown in Table 4. There were again no indications of significant block effects on any of the measures ( $p>.1$ for $\delta+1 / \beta$, and $p>.3$ for the other three parameters). Furthermore, the power to detect processing time differences was larger in this experiment than in the first one, because the interindividual variability in the block effect on $1 / \beta$ was smaller.

\section{GENERAL DISCUSSION}

In two SAT experiments, we compared response accuracy $\left(d^{\prime}\right)$ as a function of RT for blocks of trials with constant versus variable RS lags, contrasting conditions in which the participants did or did not know in advance how much time would be available for processing. In both experiments, the participants' mean response latency and accuracy depended more strongly on RS lag in the blocks with constant lags than in the blocks with variable ones, indicating that the participants were sensitive to the manipulation of constant versus variable lags. From a methodological perspective, this pattern suggests that the constant lag procedure produces larger effective shifts in the criteria underlying speed-accuracy trade-offs than does the variable lag procedure, which provides one reason to prefer the former procedure in studies of SAT phenomena.

Table 3

Percentage of Correct Responses (PC), Mean Reaction Time From Stimulus Onset (RT), Mean Reaction Time From Response Signal Onset $\left(R_{\mathrm{RS}}\right)$, and Standard Deviation of RT and $R T_{\mathrm{RS}}(S D)$ As a Function of Block Type and Response Signal (RS) Lag in Experiment 2

\begin{tabular}{|c|c|c|c|c|c|c|c|c|c|c|c|c|}
\hline \multirow{3}{*}{$\begin{array}{c}\text { Dependent } \\
\text { Variable }\end{array}$} & \multicolumn{12}{|c|}{ Block Type } \\
\hline & \multicolumn{6}{|c|}{ Variable RS Lag (msec) } & \multicolumn{6}{|c|}{ Constant RS Lag (msec) } \\
\hline & 75 & 175 & 300 & 450 & 900 & 1,800 & 75 & 175 & 300 & 450 & 900 & 1,800 \\
\hline $\mathrm{PC}$ & 62 & 73 & 86 & 93 & 93 & 95 & 57 & 71 & 87 & 93 & 94 & 94 \\
\hline RT (msec) & 354 & 412 & 505 & 642 & 1,098 & 1,997 & 309 & 385 & 496 & 637 & 1,091 & 2,000 \\
\hline $\mathrm{RT}_{\mathrm{RS}}(\mathrm{msec})$ & 279 & 237 & 205 & 192 & 198 & 197 & 234 & 210 & 196 & 187 & 191 & 200 \\
\hline$S D(\mathrm{msec})$ & 44 & 41 & 36 & 32 & 27 & 24 & 45 & 46 & 44 & 39 & 29 & 30 \\
\hline
\end{tabular}




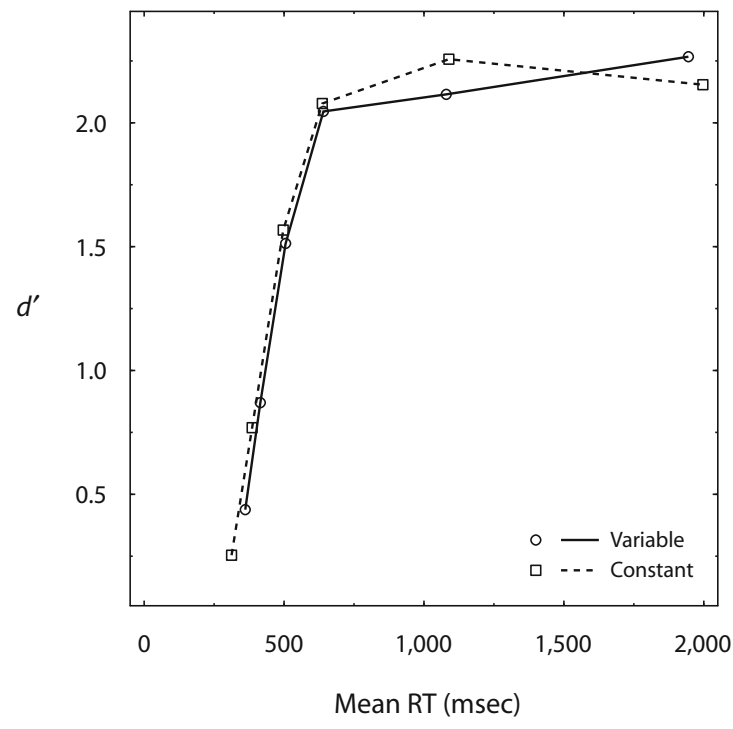

Figure 3. Response accuracy $\left(d^{\prime}\right)$ as a function of mean reaction time (RT) in constant and variable blocks in Experiment 2. The standard errors of the $d^{\prime}$ and mean RT values are 0.12 units and $6.1 \mathrm{msec}$, respectively, based on pooled error terms for both main effects and the interaction in ANOVAs with factors of block and stimulus onset asynchrony.

More critically, essentially superimposed SAT functions were obtained with constant and variable RS lags, indicating that processing is no more efficient when the processing time is known in advance than when it is not. This finding supports models in which participants respond strategically to speed pressure by changing only their criteria for the amount of evidence needed to respond, not the nature of the evidence accumulation process per se. This conclusion is consistent with the view that the processes responsible for discrimination performance are driven exclusively by bottom-up information accumulation processes (e.g., Ratcliff, 2006) and that they are not sensitive to top-down strategic modulations capable of differentially optimizing processing for a particular processing time.

The present results place clear limits on the types of strategy effects that underlie performance in SAT paradigms. The results are consistent with the common idea that participants strategically adjust a speed-accuracy criterion - by which is meant a parameter controlling the amount of evidence that must be accumulated before the response is emitted (e.g., Luce, 1986; Pachella, 1974; Ratcliff, 2006; Ruthruff, 1996; Schouten \& Bekker, 1967). Evidently, however, that is all that they adjust strategically, at least in perceptual discrimination tasks. There is no evidence that they can choose different strategies that are differentially effective for short versus long processing times, thus weakening the argument that the RS procedure should be preferred because it eliminates the possibility that processing strategy varies with processing time (Dosher, 1976; Reed, 1976). In addition, these results are consistent with models in which perceptual discriminations are made on the basis of a simple accu- mulation of sensory information (e.g., Luce, 1986), with no differential strategic selection of different types of information that might accumulate at different rates. This is not to deny the importance of other types of strategic preparation, however, because there is good evidence that perceptual processes can be modulated so as to prepare in advance to increase sensitivity to certain types of stimuli or stimulus features (e.g., Egeth \& Smith, 1967; Pachella, 1975; Sanocki, 1987)

The possibility also remains open that differences in processing efficiency between constant and variable blocks might emerge in other situations. For example, they might emerge with other types of tasks, especially those that are more heavily influenced by participant strategies. Strategic variations have been documented in a wide variety of cognitive domains (see Strayer \& Kramer, 1994a), including visual search (e.g., Corcoran \& Jackson, 1977; Gathercole \& Broadbent, 1984; Smilek, Enns, Eastwood, \& Merikle, 2006) and dual-task performance (e.g., De Jong \& Sweet, 1994; Logan \& Gordon, 2001). If different strategies are optimal under different levels of speed stress, such tasks would seem quite likely to produce more efficient processing with constant, rather than variable, RS lags (see Kleinsorge, 2001; Svenson \& Benson, 1993). As another example, differences between constant and variable blocks might emerge after more practice - even with perceptual discrimination tasks - if it is difficult for participants to discover or implement the sorts of special strategies that might be optimal for a given amount of processing time.

The present findings are consistent with research on temporal preparation suggesting that brief constant and variable RS lags $(<300 \mathrm{msec})$ should not produce a differential effect on SAT functions. For example, Bertelson and Tisseyre (1968) examined the effect of variable versus constant foreperiods on choice RT. After a predictable (constant condition) or unpredictable (variable condition) foreperiod, a visual stimulus called for a choice reaction. In the variable condition, the length of the foreperiod varied randomly from trial to trial. In the constant condition, however, foreperiod length was kept constant within a block of trials but was varied from block to block. The time course of temporal preparation on RT was similar in these two conditions, although both conditions produced a strong effect of foreperiod on RT. Hence, the results reported by Bertelson and Tisseyre suggest that strategies based on temporal preparation do not strongly affect RT with brief preparation intervals. On the other hand, there

Table 4

Average Parameter Estimates for the Model Represented by Equation 1 As a Function of Block Type in Experiment 2

\begin{tabular}{lccc}
\hline & \multicolumn{3}{c}{ Block Type } \\
\cline { 2 - 4 } \multicolumn{1}{c}{ Parameter } & RS Lag & $\begin{array}{c}\text { Constant } \\
\text { RS Lag }\end{array}$ & $\begin{array}{c}S D \text { of } \\
\text { Block Effect }\end{array}$ \\
\hline$\delta(\mathrm{msec})$ & 333 & 327 & 25.85 \\
$\lambda$ & 2.50 & 2.47 & 0.30 \\
$\beta(\mathrm{msec})$ & 168 & 150 & 61.68 \\
$\delta+1 / \beta(\mathrm{msec})$ & 501 & 477 & 48.30 \\
\hline
\end{tabular}

Note-RS, response signal; $S D$, standard deviation: $\lambda$, asymptotic value of $d^{\prime}$, a unitless quantity. 
does seem to be a contradiction between the present results and those of previous temporal preparation studies with long preparation intervals (i.e., >300 msec). As was noted in the introduction, such studies have shown faster responses with constant long preparation intervals than with variable ones, yet there was no difference between constant and variable RS lags with the two longest RS lags in the present Experiment 2. Further research will be needed to determine which of the many procedural differences between these two types of studies are responsible for the discrepancy between the present results with long RS lags and those of temporal preparation studies with long preparation intervals.

Although we have concentrated in this article on the deadline and RS procedures, it is of interest to consider the implications of the present results for several other procedures that have been used for tracing out SAT functions. Specifically, participants may be instructed to be especially fast or accurate (e.g., Band, Ridderinkhof, \& van der Molen, 2003), they may be given monetary payoffs emphasizing response speed versus accuracy (e.g., Dickman \& Meyer, 1988), or they may be required to respond within a certain time band after stimulus onset (e.g., Snodgrass, Luce, \& Galanter, 1967). For the present purposes, these alternative procedures may be regarded as variants of the deadline method, because in all of these procedures the desired processing time can be determined in advance of the trial. In contrast, Schouten and Bekker (1967) used a fixed 210-msec sequence of three tone pips as the RS, with participants instructed to respond in synchrony with the third pip. This procedure is intermediate between the traditional deadline and the RS procedures, because although the sequence started at an unpredictable time, participants did have some advance notice (approximately $200 \mathrm{msec}$ ) of when the response would be required. Thus, on the basis of the present results, we would expect no large differences among the SAT functions obtained with these procedures either.

In conclusion, we note that, methodologically, the present results suggest that there is very little difference between the deadline procedure and the RS procedure-at least with moderately practiced participants in a perceptual discrimination task - so researchers can use whichever one is more convenient in their particular circumstances. The deadline procedure has the slight advantage that it produces wider variation in mean RTs and accuracies across RS lags, so it constitutes a somewhat stronger method of manipulating SATs. On the other hand, researchers preferring the RS method could compensate for this advantage just by increasing the range of RS lags. In contrast, the RS procedure has the advantage that it produces less variable RTs, which provides a methodological argument for using variable RS lags to increase power, but this advantage is quantitatively so small that it would seem negligible in practice.

\section{AUTHOR NOTE}

This research was supported by a grant from the Marsden Fund, administered by the Royal Society of New Zealand. The authors thank Brian McElree for advice on data analysis and Charles Liu, Eric Ruthruff, and an anonymous reviewer for constructive comments on ear- lier versions of the manuscript. Correspondence concerning this article should be addressed to J. Miller, Department of Psychology, University of Otago, Dunedin, New Zealand, or R. Ulrich, Department of Cognitive and Biological Psychology, University of Tübingen, Friedrichstr. 21, 72072 Tübingen, Germany (e-mail: miller@psy.otago.ac.nz or ulrich@ uni-tuebingen.de).

\section{REFERENCES}

BAND, G. P. H., RIDDERINKHOF, K. R., \& VAN DER MOLEN, M. W. (2003). Speed-accuracy modulation in case of conflict: The roles of activation and inhibition. Psychological Research, 67, 266-279.

Bertelson, P., \& Tisseyre, F. (1968). The time-course of preparation with regular and irregular foreperiods. Quarterly Journal of Experimental Psychology, 20, 297-300.

Bonnel, A.-M., \& Hafter, E. R. (1998). Divided attention between simultaneous auditory and visual signals. Perception \& Psychophysics, 60, 179-190.

Carrasco, M., Giordano, A. M., \& McElree, B. (2004). Temporal performance fields: Visual and attentional factors. Vision Research, 44, 1351-1365.

Carrasco, M., \& McElree, B. (2001). Covert attention accelerates the rate of visual information processing. Proceedings of the National Academy of Sciences, 98, 5363-5367.

Corbett, A. T., \& Wickelgren, W. A. (1978). Semantic memory retrieval: Analysis by speed accuracy tradeoff functions. Quarterly Journal of Experimental Psychology, 30, 1-15.

Corcoran, D. W. J., \& JACKSON, A. (1977). Basic processes and strategies in visual search. In S. Dornic (Ed.), Attention and performance $V I$ (pp. 387-411). Hillsdale, NJ: Erlbaum.

De Jong, R., \& SweEt, J. B. (1994). Preparatory strategies in overlappingtask performance. Perception \& Psychophysics, 55, 142-151.

DiCKMAN, S. J., \& MEYER, D. E. (1988). Impulsivity and speed-accuracy tradeoffs in information processing. Journal of Personality \& Social Psychology, 54, 274-290.

Dosher, B. A. (1976). The retrieval of sentences from memory: A speed-accuracy study. Cognitive Psychology, 8, 291-310.

Dosher, B. A. (1982). Effect of sentence size and network distance on retrieval speed. Journal of Experimental Psychology: Learning, Memory, \& Cognition, 8, 173-207.

Dosher, B. A., HAN, S., \& Lu, Z.-L. (2004). Parallel processing in visual search asymmetry. Journal of Experimental Psychology: Human Perception \& Performance, 30, 3-27.

Dror, I. E., Busemeyer, J. R., \& Basola, B. (1999). Decision making under time pressure: An independent test of sequential sampling models. Memory \& Cognition, 27, 713-725.

Egeth, H. [E.], \& Sмith, E. E. (1967). Perceptual selectivity in a visual recognition task. Journal of Experimental Psychology, 74, 543-549.

Gathercole, S. E., \& Broadbent, D. E. (1984). Combining attributes in specified and categorized target search: Further evidence for strategic differences. Memory \& Cognition, 12, 329-337.

Gopher, D., Armony, L., \& Greenshpan, Y. (2000). Switching tasks and attention policies. Journal of Experimental Psychology: General, 129, 308-339.

Green, D. M., \& LucE, R. D. (1973). Speed-accuracy trade off in auditory detection. In S. Kornblum (Ed.), Attention and performance IV (pp. 547-569). New York: Academic Press.

KLEINSORGE, T. (2001). The time course of effort mobilization and strategic adjustments of response criteria. Psychological Research, 65, 216-223.

LeE, M. D., \& Cummins, T. D. R. (2004). Evidence accumulation in decision making: Unifying the "take the best" and the "rational" models. Psychonomic Bulletin \& Review, 11, 343-352.

Lien, M.-C., Ruthruff, E., Remington, R. W., \& Johnston, J. C. (2005). On the limits of advance preparation for a task switch: Do people prepare all the task some of the time or some of the task all the time? Journal of Experimental Psychology: Human Perception \& Performance, 31, 299-315.

LINK, S. W. (1971). Applying RT deadlines to discrimination reaction time. Psychonomic Science, 25, 355-358.

Link, S. W., \& Tindall, A. D. (1971). Speed and accuracy in comparative judgments of line length. Perception \& Psychophysics, 9, 284-288. 
LOGAN, G. D., \& GoRDON, R. D. (2001). Executive control of visual attention in dual-task situations. Psychological Review, 108, 393-434.

Low, K. A., Larson, S. L., Burke, J., \& Hackley, S. A. (1996). Alerting effects on choice reaction time and the photic eyeblink reflex. Electroencephalography \& Clinical Neurophysiology, 98, 385-393.

LuCE, R. D. (1986). Response times: Their role in inferring elementary mental organization. Oxford: Oxford University Press.

Macmillan, N. A., \& Creelman, C. D. (1991). Detection theory: A user's guide. Cambridge: Cambridge University Press.

Massaro, D. W., \& Warner, D. S. (1977). Dividing attention between auditory and visual perception. Perception \& Psychophysics, 21, 569-574.

Mattes, S., \& Ulrich, R. (1997). Response force is sensitive to the temporal uncertainty of response stimuli. Perception \& Psychophysics, 59, 1089-1097.

McElree, B., \& Carrasco, M. (1999). The temporal dynamics of visual search: Evidence for parallel processing in feature and conjunction searches. Journal of Experimental Psychology: Human Perception \& Performance, 25, 1517-1539.

McElree, B., \& Dosher, B. A. (1993). Serial retrieval processes in the recovery of order information. Journal of Experimental Psychology: General, 122, 291-315.

Niemi, P., \& NäÄTÄNEN, R. (1981). Foreperiod and simple reaction time. Psychological Bulletin, 89, 133-162.

PACHELla, R. G. (1974). The interpretation of reaction time in informationprocessing research. In B. H. Kantowitz (Ed.), Human information processing: Tutorials in performance and cognition (pp. 41-82). Hillsdale, NJ: Erlbaum.

Pachella, R. G. (1975). The effect of set on the tachistoscopic recognition of pictures. In P. M. A. Rabbitt \& S. Dornic (Eds.), Attention and performance V (pp. 137-156). New York: Academic Press.

Pachella, R. G., Fisher, D. F., \& Karsh, R. (1968). Absolute judgments in speeded tasks: Quantification of the trade-off between speed and accuracy. Psychonomic Science, 12, 225-226.

Pachella, R. G., \& Pew, R. W. (1968). Speed-accuracy tradeoff in reaction time: Effect of discrete criterion times. Journal of Experimental Psychology, 76, 19-24.

RATCLIFF, R. (2006). Modeling response signal and response time data Cognitive Psychology, 53, 195-237.

RatCLIFF, R., \& McKoon, G. (1989). Similarity information versus relational information: Differences in the time course of retrieval. Cognitive Psychology, 21, 139-155.

RatCliff, R., \& Rouder, J. N. (2000). A diffusion model account of masking in two-choice letter identification. Journal of Experimental Psychology: Human Perception \& Performance, 26, 127-140.

ReED, A. V. (1973). Speed-accuracy trade-off in recognition memory. Science, 181, 574-576.

REED, A. V. (1976). List length and the time-course of recognition in immediate memory. Memory \& Cognition, 4, 16-30.

Rinkenauer, G., Osman, A., Ulrich, R., Müller-Gethmann, H., \& MatTeS, S. (2004). On the locus of speed-accuracy tradeoff in reaction time: Inferences from the lateralized readiness potential. Journal of Experimental Psychology: General, 133, 261-282.

Rolke, B., \& HofmanN, P. (2007). Temporal uncertainty degrades perceptual processing. Psychonomic Bulletin \& Review, 14, 522-526.

Ruthruff, E. (1996). A test of the deadline model for speed-accuracy tradeoffs. Perception \& Psychophysics, 58, 56-64.
SANOCKI, T. (1987). Visual knowledge underlying letter perception: Font-specific, schematic tuning. Journal of Experimental Psychology: Human Perception \& Performance, 13, 267-278.

Schouten, J. F., \& BekKer, J. A. M. (1967). Reaction time and accuracy. Acta Psychologica, 27, 143-153.

Smilek, D., Enns, J. T., Eastwood, J. D., \& Merikle, P. M. (2006). Relax! Cognitive strategy influences visual search. Visual Cognition, 14, 543-564.

Snodgrass, J. G., Luce, R. D., \& Galanter, E. (1967). Some experiments on simple and choice reaction time. Journal of Experimental Psychology, 75, 1-17.

Stoffels, E. J., van der Molen, M. W., \& Keuss, P. J. G. (1985). Intersensory facilitation and inhibition: Immediate arousal and location effects of auditory noise on visual choice reaction time. Acta Psychologica, 58, 45-62.

Strayer, D. L., \& Kramer, A. F. (1994a). Strategies and automaticity: I. Basic findings and conceptual framework. Journal of Experimental Psychology: Learning, Memory, \& Cognition, 20, 318-341.

Strayer, D. L., \& Kramer, A. F. (1994b). Strategies and automaticity: II. Dynamic aspects of strategy adjustment. Journal of Experimental Psychology: Learning, Memory, \& Cognition, 20, 342-365.

Svenson, O., \& Benson, L., III (1993). On experimental instructions and the inducement of time pressure behavior. In O. Svenson \& A. J. Maule (Eds.), Time pressure and stress in human judgment and decision-making (pp. 157-165). New York: Plenum.

Tulving, E., \& LindSAY, P. H. (1967). Identification of simultaneously presented simple visual and auditory stimuli. Acta Psychologica, 27, 101-109.

Wickelgren, W. A., \& Corbett, A. T. (1977). Associative interference and retrieval dynamics in yes-no recall and recognition. Journal of Experimental Psychology: Human Learning \& Memory, 3, 189-202.

Wood, C. C., \& JenNings, J. R. (1976). Speed-accuracy tradeoff functions in choice reaction time: Experimental designs and computational procedures. Perception \& Psychophysics, 19, 92-102.

\section{NOTES}

1. The traditional deadline procedure does not use any RSs, so the participant is implicitly required to monitor the passage of time after stimulus onset (e.g., Pachella et al., 1968). We used RSs in both conditions to eliminate this requirement and, thereby, focus exclusively on the effects of advance knowledge.

2. Note that the within-condition standard deviations of RT and $\mathrm{RT}_{\mathrm{RS}}$ are necessarily equal, because $\mathrm{RT}$ and $\mathrm{RT}_{\mathrm{RS}}$ differ by a constant.

3. Because $\mathrm{RT}_{\mathrm{RS}}$ is a simple transformation of RT (i.e., $\mathrm{RT}_{\mathrm{RS}}=\mathrm{RT}-$ lag), the ANOVAs for these two dependent variables are closely related. With respect to the effect of RS lag under consideration here, for example, the $F$ s for the two variables necessarily have the same error term, although the effect sizes are different because lags are included in RT, but not in $\mathrm{RT}_{\mathrm{RS}}$. For other factors (e.g., block type), the $F \mathrm{~s}$ for the two variables are necessarily identical. To ease the reader's task in understanding the results presented in this article, we will simply report the $F \mathrm{~S}$ for both of these dependent variables separately, ignoring the relations between them.

(Manuscript received July 10, 2007; revision accepted for publication February 3, 2008.) 